\title{
3 Research Square

\section{A Resting-State Functional MRI Study in Patients with Vestibular Migraine During Interictal Period}

\section{Shuqing Wang}

The Affiliated Hospital of Qingdao University

Haiping Wang ( $1157318363 @ q q . c o m$ )

Department of Neurology, The Affiliated Hospital of Qingdao University

\section{Xuejun Liu}

The Affiliated Hospital of Qingdao University

\section{Wenjing Yan}

the affiliated hospital of Qingdao university

\section{Minghui Wang}

the affiliated hospital of Qingdao university

\section{Renliang Zhao}

the affiliated hospital of Qingdao university

\section{Research article}

Keywords: vestibular migraine, resting-state functional magnetic resonance imaging, vestibular processing, cerebellum

Posted Date: September 22nd, 2020

DOI: https://doi.org/10.21203/rs.3.rs-74789/v1

License: (c) (i) This work is licensed under a Creative Commons Attribution 4.0 International License. Read Full License

Version of Record: A version of this preprint was published at Acta Neurologica Belgica on March 8th, 2021. See the published version at https://doi.org/10.1007/s13760-021-01639-9. 


\section{Abstract}

Aim

To evaluate the spontaneous neuronal activities and the changes of brain functional network in patients with vestibular migraine (VM) using resting-state functional MRI (fMRI) during the interictal period.

\section{Methods}

Three groups included 18 patients with VM, 21 patients with MWoA and $21 \mathrm{HCs}$ underwent the scanning of the resting-state fMRI. First, brain regions with significant differentia of amplitude of low frequency fluctuation (ALFF) values were obtained. Secondly, functional connectivity (FC) analysis was performed in the brain region(s) with the most significant differentia of ALFF values which was defined as region of interest (ROI).

Results

(1) Compared with healthy volunteers, patients with VM and patients with MWoA showed significant ALFF decrease in the right putamen $(P<0.05)$, and significant ALFF increase in the right lingual gyrus $(P<0.05)$. What's more, compared with patients with MWoA, patients with VM showed significant ALFF increase in the right lingual gyrus $(P<0.05)$. In addition, we found that ALFF values in the right putamen of patients with VM were negatively correlated with the duration of migraine and the frequency of migraine attacks $(\mathrm{P}<0.05)$. (2) Compared with $\mathrm{HCs}$, patients with VM showed significant $\mathrm{FC}$ increase among the cerebellum, the left dorsolateral superior frontal gyrus and the right putamen $(P<0.05)$ but significant decrease among the left median cingulate, paracingulate gyri and the right putamen $(P<0.05)$. Compared with patients with MWoA, patients with VM showed significant $F C$ increase between the cerebellum and the right putamen $(P<0.05)$ but significant $F C$ decrease among the left median cingulate, paracingulate gyri and the right putamen $(P<0.05)$.

Conclusion

There are functional abnormalities in nociceptive, vestibular and visual cortex regions in patients with VM during the interictal period.

\section{Introduction}

Dizziness and headache are the most common symptoms in neurology clinics, and large epidemiologic data indicate that migraine is inextricably linked to vestibular symptoms (Von and Lempert, 2016). The International Headache Society and the International Bárány Society jointly developed and published a unified concept of "vestibular migraine" and its diagnostic criteria in 2012 (Lempert et al., 2012), which was included in the appendix of the 3rd edition of the International Classification of Headache Disorders (ICHD-3) in 2018(Headache Classification Committee of the International Headache Society, 2018). 
Vestibular symptoms that are causally related to migraine have been expressed in vestibular migraine (VM) which has become the recognized professional term (Obermann, 2017). Currently, the pathophysiology hypothesis of VM is based on knowledge of migraine. VM is increasingly thought to be a central nervous system disorder (Power et al., 2018). Multiple studies have shown that the pathophysiology of VM is importantly related to the interaction between nociceptive and vestibular system (Espinosa-Sanchez and Lopez-Escamez, 2015).

Neuroimaging techniques help to explore the pathogenesis of VM. Using voxel-based morphometry (VBM), several studies have shown that VM patients have abnormal volume of gray matter (GM) in the nociceptive and multisensory vestibular brain areas (Messina et al., 2017; Obermann et al., 2014). Moreover, several functional brain MRI studies have shown differential activation of brain regions in patients with VM during cold-water ear irrigation or visual stimulation (Russo et al., 2014; Teggi et al., 2016).

In this exploratory study, we assessed the patterns of regional brain spontaneous neuronal activity alterations and corresponding brain circuit changes in patients with VM without any stimulation during interictal period in comparison to migraine patients without aura and healthy controls (HCs). We also investigated the correlations between functional abnormalities and patients' clinical characteristics.

\section{Materials And Methods}

\section{Subjects}

We recruited 39 right-handed patients ( $18 \mathrm{VM}$, of which 3 had a past history of migraine with vestibular aura, and 21 migraine without aura (MWoA) patients) from the outpatient clinic of neurology department of our hospital from September 2018 to May 2019. All patients were enrolled according to the diagnostic criteria of ICHD-3. The enrolled patients with MWoA had no vestibular symptoms and patients with VM were enrolled according to the definite criteria for VM. The subjects were aged between 18 and 60 years. All patients were in attack-free state for three days prior to MRI scanning, the day of MRI scanning, and three days after MRI scanning (Wang et al., 2019). In addition, they were not taking any medications to prevent migraine, vertigo or dizziness. The control group consisted of 21 healthy right-handed subjects with no significant differences in gender, age, or educational level from the enrolled patients. The HCs had no pathologic pain, including chronic pain, and no family history of migraine. None of the research subjects reported other neurological, psychiatric, endocrine, cardio-cerebrovascular diseases and other major system diseases. All subjects did not have peripheral vestibular dysfunction according to videonystagmography (VNG) recordings with caloric testing. In addition, participants who abused substances (such as alcohol, nicotine) that affected test results were excluded.

No brain structural abnormalities, such as T2 hyperintensities in deep white matter, were found in the enrolled subjects through conventional MRI scanning.

\section{MRI acquisition}


All subjects whose heads were fixed with sponge pads were supine on a 3.0T GE scanner, and a conventional eight-channel quadrature head coil was used for MRI scanning to obtain images. In order to avoid the adverse effect of machine noise on the results, subjects needed to insert earplugs in their ears. In addition, subjects were required to stay awake and close their eyes without systematic thinking.

Prior to the functional run, the brain structural images of each subject were generated by a threedimensional T1-weighted magnetization prepared rapid gradient echo (3D T1-MPRAGE) sequence with following parameters: repetition time $(T R)=5.5 \mathrm{~ms}$, echo time $(T E)=1.8 \mathrm{~ms}$, flip angle $=9^{\circ}$, slice thickness $=1.0 \mathrm{~mm}, 256$ slices, matrix size $=256 \times 256$, voxel size $=0.9 \mathrm{~mm} \times 0.9 \mathrm{~mm} \times 0.9 \mathrm{~mm}$, field of view $(F O V)=256 \mathrm{~mm} \times 256 \mathrm{~mm}$. Two experienced radiologists examined all participants' brain structure information to rule out the possibility of subclinical lesions.

Next, functional blood-oxygen level-dependent (BOLD) signals were obtained using an echo-planar imaging sequence with following parameters: $T R=2000 \mathrm{~ms}, T E=35 \mathrm{~ms}$, flip angle $=90^{\circ}$, slice thickness $=3.5 \mathrm{~mm}, \mathrm{FOV}=256 \mathrm{~mm} \times 256 \mathrm{~mm}$, matrix size $=64 \times 64,64$ slices.

\section{Data preprocessing}

Statistical Parametric Mapping 12 (SPM12) software was used for data preprocessing and statistical analyses. The first 10 volumes of each subject's scanned image were discarded for subjects' adaptation to the scanning environment and scanner calibration (Hoptman et al., 2010). Then the remaining volumes were corrected for acquisition delay between slices and aligned to the first image of each session for motion correction (Xue et al., 2013). The functional image data of subjects were imported into DARTEL standard space template for spatial normalization with a resampling voxel size of $3 \mathrm{~mm} \times 3 \mathrm{~mm} \times 3 \mathrm{~mm}$. The subjects' head should not move more than $1 \mathrm{~mm}$ or rotate $1^{\circ}$ in any direction. Next, the 6 -mm fullwidth at half maximum Gaussian kernel was used to spatially smooth the images in order to improve the signal to noise ratio of the functional images and reduce the influence of noise.

Finally, imaging data were temporally filtered (band pass, $0.01-0.08 \mathrm{~Hz}$ ) to obtain the low-frequency resting-state functional magnetic resonance signal (Xue et al., 2013).

\section{ALFF analysis}

Amplitude of low frequency fluctuation (ALFF) analyses were performed by using RESTplus V1.22 software. Fast Fourier transform (FFT) was used to transform the preprocessed filtered time series into frequency domain to obtain the power spectrum. The averaged square root of each voxel in the range of $0.01-0.08 \mathrm{~Hz}$ was taken as the ALFF value. For standardization, the ALFF of each voxel was divided by the global average of the ALFF value (Xue et al., 2013).

\section{ROI-based FC analysis}

Before performing functional connectivity $(\mathrm{FC})$ analysis, multiple sources of false variance were removed from the preprocessed data by linear regression, including head motion parameters, global average BOLD signals and average BOLD signals of ventricular and white matter regions. Region of interest (ROI) 
method was adopted for FC analysis. The brain region with the largest differentia in ALFF comparison was selected as ROI to calculate the whole brain and its FC, and fisher-z transformation was conducted to obtain the FC map of each subject. Finally, analysis of covariance was used to quantitatively compare the FC of brain regions in the three groups.

\section{Statistics}

The data of demographics and clinical characteristics were expressed by $\bar{\chi} \pm s$ and percentage. Fisher exact test was used to analyze the difference among the categorical data, and at the same time, student's t-test and analysis of variance were used to analyze the difference of the continuous variables. These data were analyzed using SPSS 22.0.

The comparisons of ALFF and FC maps which had smoothed among three groups were assessed using analysis of covariance in SPM12, including age and sex as covariates. At the same time, ALFF survived with FDR correction. Then, the Bonferroni method was used for multiple comparisons, and the P-value threshold was less than 0.05 (double tails). Finally, the results were displayed with xjView and MRIcron software.

The ALFF values of the VM group corresponding to the ROI were extracted and correlated with the clinical characteristics of patients with VM. $\mathrm{P}<0.05$ was considered statistically significant.

\section{Results}

Demographics and clinical characteristics are summarized in Table 1. There was no difference among the three groups in terms of age $(p=0.915)$, gender $(p=0.922)$, and education level $(p=0.954)$.

Table 1

Demographics and patient characteristics

\begin{tabular}{|llll|}
\hline & VM & MWoA & HCs \\
\hline Number of subjects & 18 & 21 & 21 \\
\hline Age (years) & $36.17 \pm 8.65$ & $36.81 \pm 11.61$ & $36.15 \pm 12.11$ \\
\hline Gender (male/female) & $3 / 15$ & $4 / 17$ & $5 / 16$ \\
\hline education level (years) & $12.11 \pm 2.47$ & $11.86 \pm 2.74$ & $12.00 \pm 2.51$ \\
\hline VM disease duration (years) & $6.56 \pm 3.36$ & NA & NA \\
\hline Migraine disease duration (years) & $12.67 \pm 7.62$ & $11.57 \pm 7.32$ & NA \\
\hline Attack frequency per month & $2.72 \pm 2.23$ & $1.86 \pm 1.20$ & NA \\
\hline NA: not applicable; VM: vestibular migraine; MWoA: migraine without aura; HCs: healthy controls. \\
\hline
\end{tabular}


The results of ALFF analysis among the three groups by analysis of covariance are summarized in Table 2 and shown in Fig. 1. The ALFF values of right putamen and right lingual gyrus were not completely equal ( $p<0.05$, FDR corrected, cluster extent $=10$ voxels). The results of the Bonferroni multiple comparisons are shown in Table 3. Compared with healthy control subjects, the patients with VM and the patients with MWoA showed significant ALFF decrease in the right putamen $(P<0.05)$, and significant increase in the right lingual gyrus $(P<0.05)$. What's more, compared with MWoA patients, patients with VM showed significant ALFF increase in the right lingual gyrus $(P<0.05)$. In addition, we found that ALFF values in the right putamen was negatively correlated with the duration of migraine and the frequency of migraine attacks $(P<0.05)$. The results are shown in Table 4.

Table 2

Regions showing significant ALFF differences among VM patients, MWoA patients, and $\operatorname{HCs}(p<0.05$, FDR corrected, cluster extent $=10$ voxels)

\begin{tabular}{|llllll|}
\hline Brain regions (AAL) & Cluster extent (number of voxels) & \multicolumn{3}{l|}{$\begin{array}{l}\text { Peak MNI coordinates } \\
(\mathbf{X Y Z )}\end{array}$} & F Peak intensity \\
\hline Putamen_R & 38 & 30 & 12 & -9 & 15.9024 \\
Lingual_R & 11 & 27 & -54 & -3 & 14.7124 \\
\hline $\begin{array}{l}\text { AAL: Anatomical Automatic Labeling; L: left; R: right; MNI: Montreal Neurological Institute; } \\
\text { Putamen_R: right putamen; Lingual_R: right lingual gyrus. }\end{array}$ \\
\hline
\end{tabular}

Table 3

Regions showing significant ALFF differences between patients and controls as well as between different groups of migraineurs using the Bonferroni multiple comparisons $(p<0.05)$.

\begin{tabular}{|lllll|}
\hline Brain regions (AAL) & Group 1 & Group 2 & $\begin{array}{l}\text { Mean Difference } \\
\text { (Group 1 - Group 2) }\end{array}$ & P value \\
\hline Putamen_R & VM & HCs & -0.457417 & 0.000 \\
\hline Putamen_R & MWoA & HCs & -0.353476 & 0.000 \\
\hline Lingual_R & VM & HCs & 0.994779 & 0.000 \\
Lingual_R & VM & MWoA & 0.496900 & 0.030 \\
\hline Lingual_R & MWoA & HCs & 0.497879 & 0.023 \\
\hline
\end{tabular}


Table 4

The correlations between ALFF values of patients with VM in the right putamen and patients' clinical characteristics

\begin{tabular}{|llll|}
\hline & $\overline{\mathbf{X}} \pm \mathbf{s}$ & Correlation coefficient $\AA$ & $\mathbf{P}$ \\
\hline the frequency of migraine attacks & $3.62 \pm 3.48$ & -0.529 & 0.035 \\
\hline the duration of migraine & $13.25 \pm 8.31$ & -0.517 & 0.040 \\
\hline
\end{tabular}

FC of brain regions in the patients with VM and subjects in two other groups were calculated using the right putamen as the ROI. The results of FC analysis among the three groups by analysis of covariance are summarized in Table 5 and shown in Fig. 2. The FC values of the right cerebellum, the left dorsolateral superior frontal gyrus and the left median cingulate and paracingulate gyri were not completely equal $(p<0.001$, cluster extent $=5$ voxels). The results of the Bonferroni multiple comparisons are shown in Table 6. Compared with healthy volunteers, patients with VM showed significant FC increase among the right cerebellum, the left dorsolateral superior frontal gyrus and the right putamen ( $P$ $<0.05$ ) but significant FC decrease among the left median cingulate, the paracingulate gyri, and the right putamen $(P<0.05)$. Compared with MWoA patients, patients with VM showed significant $F C$ increase in the right cerebellum and in the right putamen $(P<0.05)$ but significant $F C$ decrease among the left median cingulate, the paracingulate gyri, and the right putamen $(P<0.05)$. Compared with $H C s$, patients with MWoA only showed significant FC increase in the left dorsolateral superior frontal gyrus and in the right putamen $(P<0.05)$.

Table 5

Regions showing significant FC differences among VM patients, MWoA patients, and HCs $(p<0.001$, cluster extent $=5$ voxels)

\begin{tabular}{|c|c|c|c|c|c|}
\hline Brain regions (AAL) & Cluster extent (number of voxels) & $\begin{array}{l}\text { Peak } \\
(X Y\end{array}$ & INI co & inates & F Peak intensity \\
\hline Cerebelum_6_R & 19 & 9 & -60 & -24 & 11.4059 \\
\hline Frontal_Sup_L & 7 & -15 & 63 & 24 & 10.2476 \\
\hline Cingulum_Mid_L & 5 & -12 & -42 & 48 & 9.4814 \\
\hline
\end{tabular}


Table 6

Regions showing significant FC differences between patients and controls as well as between different groups of migraineurs using the Bonferroni multiple comparisons $(p<0.05)$.

\begin{tabular}{|lllll|}
\hline Brain regions (AAL) & Group 1 & Group 2 & $\begin{array}{l}\text { Mean Difference } \\
\text { (Group 1 - Group 2) }\end{array}$ & P value \\
\hline Cerebelum_6_R & VM & HCs & 0.291095 & 0.000 \\
\hline Cerebelum_6_R & VM & MWoA & 0.240688 & 0.001 \\
\hline Frontal_Sup_L & MWoA & HCs & 0.237301 & 0.000 \\
\hline Frontal_Sup_L & VM & HCs & 0.194002 & 0.004 \\
Cingulum_Mid_L & VM & HCs & -0.189849 & 0.004 \\
\hline Cingulum_Mid_L & VM & MWoA & -0.249289 & 0.000 \\
\hline
\end{tabular}

\section{Discussion}

Resting-state functional MRI (fMRI) which is a noninvasive imaging technique measures low-frequency fluctuations in BOLD signals (Fox and Raichle, 2007). Cortical and subcortical areas of spatially distinct and functionally related groups will show related spontaneous fluctuations during the resting state, composed of intrinsic functional networks of the human brain (Seeley et al., 2009). Changes in internal functional networks will affect performance of tasks in real life (Fox et al., 2007; (Hesselmann et al., 2008). In addition, the resting-state method has been widely used to reveal the typical and atypical functional structures inherent in the brain (Fox and Raichle, 2007; Greicius, 2008). Changes in characteristics at rest can be used as indicators of the progression of various diseases, such as heroin addiction (Yuan et al., 2010; Yuan et al., 2010; Yuan et al., 2010), Alzheimer's disease (Greicius et al., 2004) and schizophrenia (Lui et al., 2009).

Most resting-state fMRI studies use FC to study temporal relationships between internal fluctuations observed in spatially distinct brain regions. However, there are few local features of spontaneous brain activity observed in specific areas by the method of FC. As a complement to the method of FC, the ALFF (Zang et al., 2007) method which measures regional spontaneous neuronal activity during resting-state fMRI is also widely used. In this study, we evaluated regional changes in both regional spontaneous neuronal activity and corresponding brain circuits during rest.

\section{ALFF}

In this study, we identified several brain regions showing differences among the three groups during the resting state. "Balance triple" refers to the visual, proprioceptive and vestibular systems that maintain the balance of the human body. The primary visual cortex, as well as the lingual gyrus and lateral geniculate body, belongs to the medial visual cortex, which is responsible for processing visual information 
(Beckmann et al., 2005; Mantini et al., 2007). This study showed that the ALFF values of lingual gyrus in patients with VM were significantly higher than that in patients with MWoA and HCs, which indicated that the spontaneous neuronal activity of lingual gyrus in patients with VM under resting state was enhanced and the excitability was increased. It may reflect the changes in the integration function of visual information in the brain of patients with $\mathrm{VM}$, which is most likely an adaptive change in response to repeated vertigo. The caudate nucleus and the lenticular nucleus are important components of the basal ganglia involved in the regulation of pain. In the study, the function of basal ganglia in the most patients with VM and MWoA was abnormal. Most scholars believe that the basal ganglia need to be involved in pain regulation due to repeated headaches, and the basal ganglia may be damaged during the process, which leads to a decrease in the ability of pain regulation. And with the increase of frequency and duration of attacks, this decline is more obvious.

FC

As one of the most commonly used analytical methods of FC, the ROI correlation analysis method has been widely used in the research of brain sensation, cognitive and emotion. In this study, the ALFF values of the three groups of subjects were calculated, and it was found that the brain area with the most significant difference was the right putamen. Therefore, it was designated as the ROI, and ALFF and FC were combined to describe the functional characteristics between brain regions. The ALFF combined with FC method has been widely used in a variety of brain diseases and provides important information for understanding these diseases, such as Alzheimer's disease (Sorg et al., 2007), heroin addiction (Yuan et al., 2010; Yuan et al., 2010; Yuan et al., 2010; Jiang et al., 2011), schizophrenia (Hoptman et al., 2010; Zhou et al., 2007) and depression (Anand et al., 2005).

The vestibular center also includes the subtentorial brainstem and cerebellar associated nucleus mass. Some scholars (Marcelli et al., 2009) have conducted fMRI studies on the vestibule of patients with cold water stimulation. They found that the brain areas with neuronal activity changes mainly included the insular cortex, thalamus, brainstem and cerebellum. The sensorimotor components associated with balance include the brainstem pathway, which produces motor responses to the body (such as the vestibule-oculomotor reflex) and visceral (the vestibular sympathetic and parasympathetic nerves) (Balaban and Yates, 2004; Holstein et al., 2011). These vestibular sensorimotor responses are regulated by cerebellum (Balaban and Yates, 2004; Ito, 1984). Patients with VM did show impaired central integration of semicircular canal rotation signal and otolithic organ gravity signal center (Wang and Lewis, 2016). Some studies have found that some patients with VM had lesions at the caudal part of the cerebellum, so the correct processing of otolitic information couldn't be carried out, and patients with VM would be highly sensitive to head movement (Espinosa-Sanchez and Lopez-Escamez, 2015). The most common abnormality of eye movement in patients with VM is central positional nystagmus(Radtke et al., 2012), which may suggest that the cerebellum inhibits the release of vestibular effect (Bronstein and Lempertt, 2007). Some studies suggested that central positional nystagmus was the result of lesions at nodules and lingual lobe of cerebellum which made vestibular maladjustment (Choi et al., 2014). Therefore, cerebellar dysfunction may be involved in the pathogenesis of VM. Previous study found that 
functional imaging at the onset of VM showed bilateral cerebellar activation (Shin et al., 2014). Cerebellar hyperactivity during the onset and interictal stages of patients with VM was considered to be an adaptive mechanism to inhibit hyperactivity of the vestibular system (Jeong et al., 2015). VM is closely linked to motion sickness (Akdal et al., 2015), and even during the interictal stages, patients with VM are less tolerant of riding a carousel, a car, or even watching a 3D movie. Changes in the functional activity of the cerebellum in patients with VM found in this study might partially explain this phenomenon, suggesting that VM was not a simple episode disease. In functional imaging studies, it was found that the brain regions involved in pain information processing mainly included the anterior cingulate cortex, prefrontal cortex, insula, cerebellum, brainstem and somatosensory cortex (Yu et al., 2011). The cerebellum, as part of the pain matrix, is involved in pain processing and regulation (Yu et al., 2011). In animals and humans, the deep cerebellar nucleus process harmful stimuli and participate in pain perception and suppression through the relationship with the brainstem nucleus and thalamus. When cerebellar activity is forcibly enhanced, the pain threshold will increase, suggesting that cerebellum's anti-nociceptive effect is enhanced (Pereira et al., 2017). The cerebellum also exhibits emotional processing functions (Klingner et al., 2013), which indicates that changes in cerebellar function can easily lead to adverse emotions. Unfavorable emotions are important factors that can trigger VM and MWoA. When the emotional function of cerebellum is abnormal, it may induce VM attacks.

Dorsolateral superior frontal gyrus is an important part of limbic system and an important part of emotional pathway of pain perception. This study found that the FC between the cerebellum, dorsolateral superior frontal gyrus and ROI of patients with VM were enhanced, and the FC between the dorsolateral superior frontal gyrus and ROI was enhanced in patients with MWoA. It suggested that patients with VM and MWoA had suffered from painful stimulation for a long time, and their endogenous analgesic mechanisms were adjusted, which might be used to process and modify painful emotional responses, or to reduce pain perception and cognition, so as to reduce input of pain signals.

Median cingulate and paracingulate gyri are not only involved in the attention and emotional response to pain, but also in the subjective perception of pain and its cognition and memory (Bluhm et al., 2007).

They are also important brain regions that form the default network (Uckner et al., 2008). In a comparative study (Liu et al., 2006) of HCs and patients with chronic pain, a high concentration of opioid receptor binding sites was observed in the cingulate gyrus, suggesting that median cingulate and paracingulate gyri played an important role in the formation and regulation of pain sensation. In this study, FC of the median cingulate and paracingulate gyri and ROI was weakened in patients with VM, which might be the result of adaptation of nervous system. The attenuation of the nervous system itself can not only reduce the pain response and emotional response, but also reduce the subjective perception of pain.

The pathogenesis of VM is not clear. Imbalance of excitement or inhibition of various sensory information, vestibular information and pain signals may lead to the occurrence of vestibular migrane (Espinosa-Sanchez and Lopez-Escamez, 2015). The study found that patients with VM had reduced activity in multiple vestibular functional areas, while other vestibular functional areas showed 
compensatory increased activity, and activity of pain-related brain areas changed similar to patients with migraine. In patients with VM, abnormal brain functional networks associated with the right putamen were observed. All of these findings are likely due to adaptive changes in the brain regions of patients with VM.

\section{Conclusions}

There are functional abnormalities in nociceptive, vestibular and visual cortex regions in patients with VM during interictal period, which not only indicates VM is not a simple paroxysmal disease, but also supports the hypothesis that VM is a central nervous system disease. These findings lead to a better understanding of the pathogenesis of VM.

\section{Declarations}

\section{Compliance with ethical standards}

Conflicts of interest This study was approved by the Local Ethical Committes on human studies and all subjects provided written informedconsent prior to study participation.

Ethical standards The study was approved by the Local Ethical Committee on human studies and was performed in accordance with the ethical standards laid down in the 1964 Declaration of Helsinki and its later amendments.

Informed consent Written informed consent was obtained from all participants at inclusion.

\section{Acknowledgements}

The authors would like to express their gratitude to Qingdao people's livelihood science and technology Project [17-3-3-18-nsh] for the financial support provided.

\section{References}

1. Von BM, Lempert T (2016) Vestibular migraine. Handb Clin Neurol 137:301-316

2. Lempert T, Olesen J, Furman J, Waterston J, Seemungal B, Carey J, Bisdorff A, Versino M, Evers S, Newman-Toker D (2012) Vestibular migraine: diagnostic criteria. J Vestib Res 22:167-172

3. Headache Classification Committee of the International Headache Society (IHS) (2018) The International Classification of Headache Disorders, 3rd edition. Cephalalgia. 38, 1-211

4. Obermann M (2017) Editorial: Vestibular migraine. Front Neurol 8:213

5. Power L, Shute W, Mcowan B, Murray K, Szmulewicz D (2018) Clinical characteristics and treatment choice in vestibular migraine. J Clin Neuro 52:50-53 
6. Espinosa-Sanchez JM, Lopez-Escamez JA (2015) New insights into pathophysiology of vestibular migraine. Front Neurol 6:1-6

7. Messina R, Rocca MA, Colombo B, Teggi R, Falini A, Comi G, Filippi M (2017) Structural brain abnormalities in patients with vestibular migraine. J Neurol 264:295-303

8. Obermann M, Wurthmann S, Steinberg BS, Theysohn N, Diener HC, Naegel S (2014) Central vestibular system modulation in vestibular migraine. Cephalalgia 34:1053-1061

9. Russo A, Marcelli V, Esposito F, Corvino V, Marcuccio L, Giannone A, Conforti R, Marciano E, Tedeschi $G$, Tessitore A (2014) Abnormal thalamic function in patients with vestibular migraine. Neurology 82:2120-2126

10. Teggi R, Colombo B, Rocca MA, Bondi S, Messina R, Comi G, Filippi M (2016) A review of recent literature on functional MRI and personal experience in two cases of definite vestibular migraine. Neurol Sci 37:1399-1402

11. Wang S, Wang H, Zhao D, Liu X, Yan W, Wang M, Zhao R,2019. Grey matter changes in patients with vestibular migraine. Clin. Radiol. 74, 898.e1-898.e5

12. Hoptman MJ, Zuo XN, Butler PD, Javitt DC, D’Angelo D, Mauro CJ, Milham MP (2010) Amplitude of low-frequency oscillations in schizophrenia: a resting state fMRI study. Schizophr Res 117:13-20

13. Xue T, Yuan K, Cheng P, Zhao L, Zhao L, Yu D, Dong T, Karen M, Gong Q, Qin W, Tian J (2013) Alterations of regional spontaneous neuronal activity and corresponding brain circuit changes during resting state in migraine without aura. NMR Biomed 26:1051-1058

14. Fox MD, Raichle ME (2007) Spontaneous fluctuations in brain activity observed with functional magnetic resonance imaging. Nat Rev Neurosci 8:700-711

15. Seeley WW, Crawford RK, Zhou J, Miller BL, Greicius MD (2009) Neurodegenerative diseases target large-scale human brain networks. Neuron 62:42-52

16. Fox MD, Snyder AZ, Vincent JL, Raichle ME (2007) Intrinsic fluctuations within cortical systems account for intertrial variability in human Behavior. Neuron 56:171-184

17. Hesselmann G, Kell CA, Eger E, Kleinschmidt A (2008) Spontaneous local variations in ongoing neural activity bias perceptual decisions. Proc. Natl. Acad. Sci. 105, 10984-10989

18. Greicius M (2008) Resting-state functional connectivity in neuropsychiatric disorders. Curr Opin Neurol 21:424-430

19. Yuan K, Qin W, Dong M, Liu J, Liu P, Zhang Y, Sun J, Wang W, Wang Y, Li Q (2010) Combining spatial and temporal information to explore restingstate networks changes in abstinent heroin-dependent individuals. Neurosci Lett 475:20-24

20. Yuan K, Qin W, Dong M, Liu J, Sun J, Liu P, Zhang Y, Wang W, Wang Y, Li Q, Zhao L, Deneen KM, Liu Y, Gold MS, Tian J (2010) Gray matter deficits and resting-state abnormalities in abstinent heroindependent individuals. Neurosci Lett 482:101-105

21. Yuan K, Qin W, Liu JX, Guo QA, Dong MH, Sun JB, Zhang Y, Liu P, Wang W, Wang YR, Li QA, Yang WC, Deneen KM, Gold MS, Liu YJ, Tian J (2010) Altered small-world brain functional networks and 
duration of heroin use in male abstinent heroin-dependent individuals. Neurosci Lett 477:37-42

22. Greicius MD, Srivastava G, Reiss AL, Menon V (2004) Default-mode network activity distinguishes Alzheimer's disease from healthy aging: evidence from functional MRI. Proc. Natl. Acad. Sci. 101, 4637-4642

23. Lui S, Deng W, Huang XQ, Jiang LJ, Ma XH, Chen HF, Zhang TJ, Li XL, Li DM, Zou L, Tang HH, Zhou XHJ, Mechelli A, Collier DA, Sweeney JA, Li T, Gong QY (2009) Association of cerebral deficits with clinical symptoms in antipsychotic-naive first-episode schizophrenia: an optimized voxel-based morphometry and resting state functional connectivity study. Am Psychiatric Assoc 166:196-205

24. Zang YF, He Y, Zhu CZ, Cao QJ, Sui MQ, Liang M, Tian LX, Jiang TZ, Wang YF (2007) Altered baseline brain activity in children with ADHD revealed by resting-state functional MRI. Brain Dev 29:83-91

25. Beckmann CF, DeLuca M, Devlin JT, Smith SM (2005) Investigations into resting-state connectivity using independent component analysis. Philos Trans R Soc Lond B Biol Sci 360:1001-1013

26. Mantini D, Perrucci MG, Del, Gratta C, Romani GL, Corbetta M (2007) Electrophysiological signatures of resting state networks in the human brain. Proc. Natl. Acad. Sci. 104, 13170-13175

27. Sorg C, RiedI V, Muhlau M, Calhoun VD, Eichele T, Laer L, Drzezga A, Forstl H, Kurz A, Zimmer C, Wohlschlager AM (2007) Selective changes of resting-state networks in individuals at risk for Alzheimer's disease. Proc. Natl. Acad. Sci. 104, 18760-18765

28. Jiang G, Qiu Y, Zhang X, Han LJ, Lv XF, Li L, Lin C, Zhuo F, Hu S, Tian J (2011) Amplitude lowfrequency oscillation abnormalities in the heroin users: A resting state fMRI study. Neuroimage $57: 149-154$

29. Zhou Y, Liang M, Tian L, Wang K, Hao Y, Liu H, Liu Z, Jiang T (2007) Functional disintegration in paranoid schizophrenia using resting-state fMRI. Schizophr Res 97:194-205

30. Anand A, Li Y, Wang Y, Wu J, Gao S, Bukhari L, Mathews VP, Kalnin A, Lowe MJ (2005) Activity and Connectivity of Brain Mood Regulating Circuit in Depression: A Functional Magnetic Resonance Study. Biol Psychiatry 57:1079-1088

31. Marcelli V, Esposito F, Aragri A, Furia T, Riccardi P, Tosetti M, Biagi L, Marciano E, Di, Salle F (2009) Spatio-temporal pattern of vestibular information processing after brief caloric stimulation. Eur $\mathrm{J}$ Radiol 70:312-316

32. Balaban C, Yates BJ (2004) Vestibulo-autonomic interactions: a teleologic perspective. In: Highstein SN, Fay RR, Popper AN (eds) Springer handbook of auditory research: the vestibular system. Springer-Verlag, New York, pp 286-342

33. Holstein GR, Friedrich VL, Kang T, Kukielka E, Martinelli GP (2011) Direct projections from the caudal vestibular nuclei to the ventrolateral medulla in the rat. Neuroscience 175:104-117

34. Ito M (1984) The cerebellum and neural control. Raven Press, New York

35. Wang J, Lewis RF (2016) Contribution of intravestibular sensory conflict to motion sickness and dizziness in migraine disorders. J Neurophysiol 116:1586-1591 
36. Radtke A, Brevern M, Neuhauser H, Hottenrott T, Lempert T (2012) Vestibular migraine: Long-term follow-up of clinical symptoms and vestibulo-cochlear findings. Neurology 79:1607-1614

37. Bronstein M, Lempertt (2007) Dizziness: A practical approach to diagnosis and management, 93. Cambridge University Press, Cambridge, pp 149-150

38. Choi JY, Kim JH, Kim HJ, Glasauer S, Kim JS (2015) Central paroxysmal positional nystagmus: Characteristics and possible mechanisms. Neurology 84:2238-2246

39. Shin JH, Kim YK, Kim HJ (2014) Altered brain metabolism in vestibular migraine: comparison of interictal and ictal findings. Cephalalgia 34:58-67

40. Jeong SH, Oh SY, Kim HJ, Koo JW, Kim JS (2010) Vestibular dysfunction in migraine: effects of associated vertigo and motion sickness. J Neurol 257:905-912

41. Akdal G, Baykan B, Karli N (2015) Population-based study of vestibular symptoms in migraineurs. Acta oto-laryngologica 135:435

42. Yu D, Yuan K, Zhao L, Zhao L, Dong M, Liu P, Wang G, Liu J, Sun J, Zhou G, Deneena K, Liang F, Qin W, Tian J (2011) Regional homogeneity abnormalities in patients with interictal migraine without aura: a resting-state study. NMR Biomed 25:806-812

43. Pereira M, Rafiq B, Chowdhury E, Babayev J, Boo H, Metwaly R, Sandilyaa P, Chusida E, Battaglia F (2017) Anodal cerebellar tDCS modulates lower extremity pain perception. NeuroRehabilitation 40:195-200

44. Klingner CM, Volk GF, Flatz C, BrodoehI S, Dieterich M, Witte OW, Guntinas-Lichius O (2013) Components of vestibular cortical function. Behav Brain Res 236:194-199

45. Bluhm RL, Miller J, Lanius RA, Osuch EA, Boksman K, Neufeld R, Theberge J, Schaefer B, Williamson P (2007) Spontaneous Low-Frequency Fluctuations in the BOLD Signal in Schizophrenic Patients: Anomalies in the Default Network. Schizophr Bull 33:1004-1012

46. Uckner RL, Andrews-Hanna JR, Schacter DL (2008) The brain's default network: anatomy, function, and relevance to disease. Ann NY Acad Sci 1124:1-38

47. Liu H, Liu Z, Liang M, Hao Y, Tan L, Kuang F, Yi Y, Xu L, Jiang T (2006) Decreased regional homogeneity in schizophrenia: a resting state functional magnetic resonance imaging study. Neuro Report 17:19-22

\section{Figures}




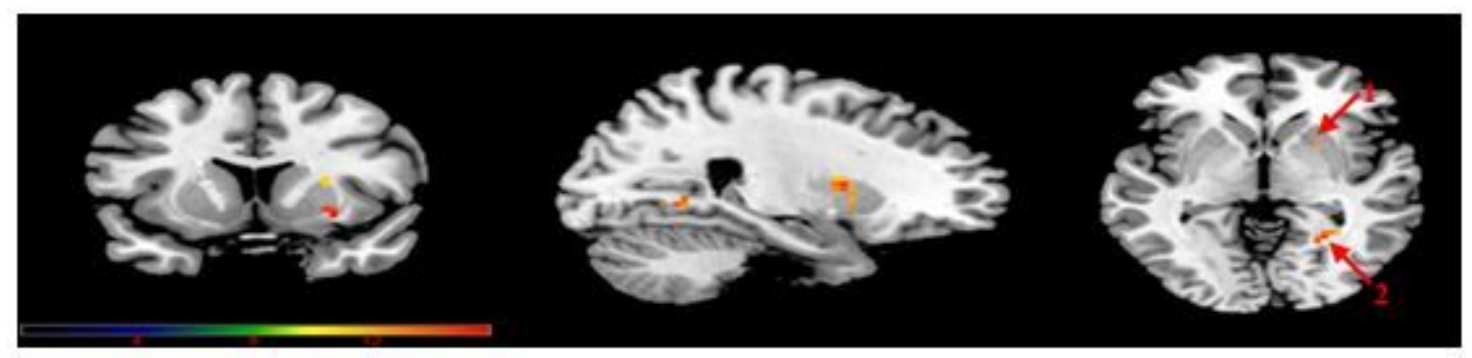

(a)

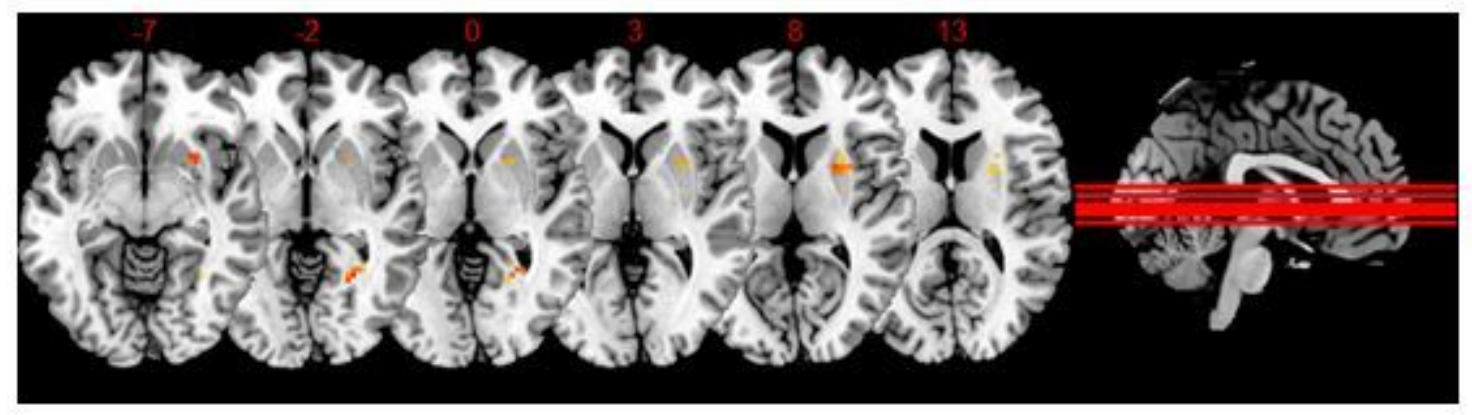

(b)

\section{Figure 1}

Areas showing significant ALFF differences ( $p<0.05$, FDR corrected, cluster extent $=10$ voxels) among VM, MWoA and HCs. Right side of the image is the right side of the brain. (a): Regions of increased ALFF were shown according to a yellow-red scale (color coded according to intensity of activation). Arrow 1: right putamen; Arrow 2: right lingual gyrus. (b): The numbers in the figure represented the number of layers in standard template space. 


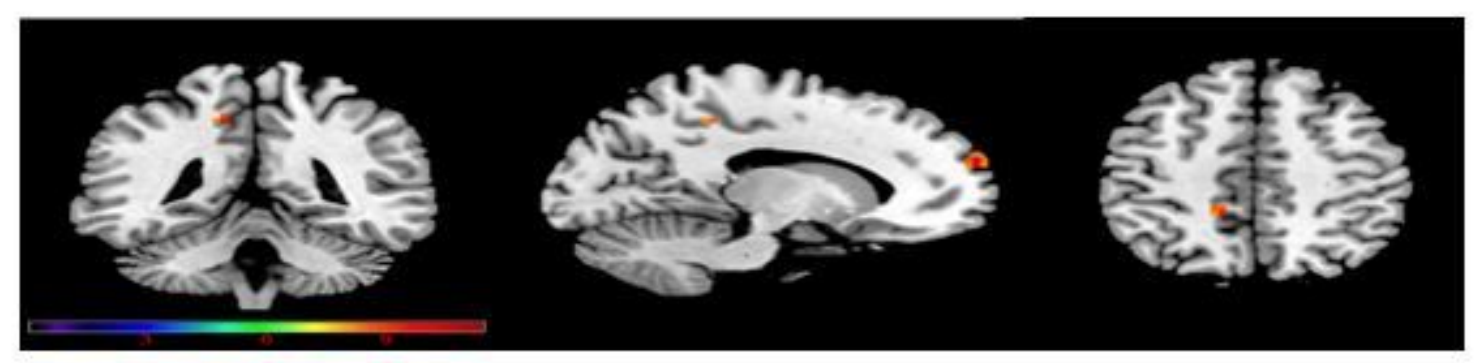

(a)
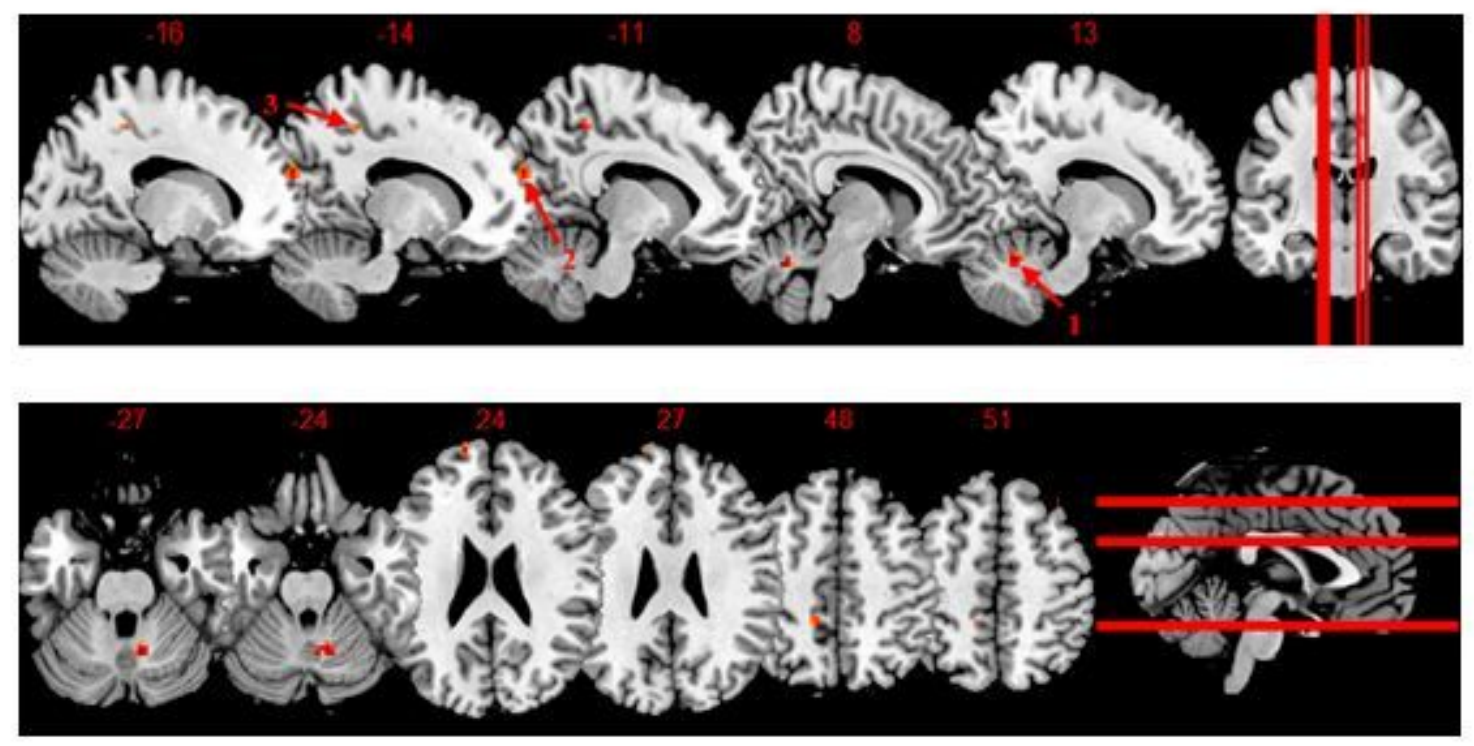

(b)

\section{Figure 2}

Areas showing significant FC differences ( $p<0.001$, cluster extent $=5$ voxels) among VM, MWoA and HCs. Right side of the image is the right side of the brain. (a): Regions of increased FC were shown according to a yellow-red scale (color coded according to intensity of activation). (b): The numbers in the figure represented the number of layers in standard template space. Arrow 1: right cerebellum; Arrow 2: left dorsolateral superior frontal gyrus; Arrow 3: left median cingulate and paracingulate gyri. 